\title{
Presence of Class I and Class II Integrons in Methicilin Resistant Staphylococci and Their Relations with Antibiotic Resistance: A Preliminary Study from Turkey
}

\author{
Defne Gumus (Corresponding author) \\ Istanbul Yeni Yuzyil University, Faculty of Medicine, \\ Department of Medical Microbiology, Istanbul, Turkey \\ E-mail: defne.gumus@yeniyuzyil.edu.tr \\ Fatma Kalayci-Yuksek \\ Istanbul Yeni Yuzyil University, Faculty of Medicine, \\ Department of Medical Microbiology, Istanbul, Turkey \\ E-mail: fatma.kalayci@yeniyuzyil.edu.tr \\ Derya Bayirli-Turan \\ Istanbul Yeni Yuzyil University, Faculty of Medicine, \\ Department of Infectious Diseases and Clinical Microbiology, Istanbul, Turkey \\ E-mail: deryabturan@gmail.com \\ Mert Caliskan \\ Istanbul Yeni Yuzyil University, Faculty of Arts and Science, \\ Department of Molecular Biology and Genetics, Istanbul, Turkey \\ E-mail: mert_34630@hotmail.com \\ Acelya Gumus \\ Istanbul Yeni Yuzyil University, Faculty of Arts and Science, \\ Department of Molecular Biology and Genetics, Istanbul, Turkey \\ E-mail: acelya.gumus.96@ hotmail.com \\ Selen Ozdemir \\ Istanbul Yeni Yuzyil University, Faculty of Arts and Science, \\ Department of Molecular Biology and Genetics, Istanbul, Turkey \\ E-mail: selensude_ozdemir@hotmail.com \\ Asli Ceren Macunluoglu \\ Uludag University, Institute of Health Sciences, \\ Department of Biostatistics, Bursa, Turkey \\ E-mail: cerenmacunluoglu@gmail.com \\ Mine Ang-Kucuker \\ Istanbul Yeni Yuzyil University, Faculty of Medicine, \\ Department of Medical Microbiology, Istanbul, Turkey \\ E-mail: mine.kucuker@yeniyuzyil.edu.tr
}

The Research Is Financed By the Scientific and Technological Research Council of Turkey

\begin{abstract}
Background: Emergence of antibiotic resistance is a major public health concern. It is known that antibiotic resistance is transferred by different ways. Integrons as one of these mechanisims cause to spread antibiotic resistance in Gram negative bacteria but also it is shown to be effective for transferring genes in Gram positive bacteria. In the present study we aimed to examine the prevalence of class I and class II integrons in MRSA and MRCNS strains isolated from patients and to determine the relationship between antibiotic resistance and the presence of integrons.
\end{abstract}


Methods: Sixty four MRSA and 62 MRCNS strains were included in this study. Antibiotic susceptibility testings were performed. Genomic and plasmid DNAs were extracted and Polymerase Chain Reaction (PCR) was used for the detection of the intI and intII genes. The PCR products were visualised in $1.5 \%$ agarose gel electrophoresis. Pearson chi-square test and Fisher's exact test were used for comparing categorical variables.

Results: Among 126 staphylococci 11 (8.7\% - 4 MRSA and 7 MRCNS) were shown to carry class I integron; whereas 7 MRCNS (5.5\%) were class II positive. Both of class I and class II integrons were detected to possess in four MRSA (3.2\%). There was no statistically significant relation between presence of integrons and resistance to each of antibiotics $(\mathrm{p}>0.05)$.

Conclusion \& Recommendation: In the present study we did not find any significant relation between resistance rates and the presence of integrons but we suggest that these results showed an important data about the extended distributions of integrons not only among Gram negative bacteria but also in staphylococci.

Keywords: MRSA, MRCNS, class I integron, class II integron, anbiotic resistance

DOI: $10.7176 / \mathrm{JHMN} / 75-07$

\section{INTRODUCTION}

Infections, especially nosocomial infections, caused by multi drug resistant bacteria continued to be a significant concern. There are two major genetic mechanisms of antibiotic resistance: 1) mutations in gene(s) and 2) acquisition of foreign DNA coding for resistance determinants through horizontal gene transfer, through three main strategies, transformation, transduction (phage mediated) and conjugation [1-5]. Horizontally transferring of genes encoding antibiotic resistance between different species or genera allows the huge dissemination of antibiotic resistance. Emergence of resistance often involves with conjugation. In conjugation, mobile genetic elements play a very important role as vehicles to share valuable genetic information. The most important mobile genetic elements are plasmids, transposons, gene cassettes and integrons [5-9].

Integrons represent the mechanism for accumulating antimicrobial resistance genes providing the addition of new genes into bacterial chromosomes, along with the necessary machinery to make their expression possible [7, 10-13]. Since Stokes \& Hall (1989) first discovered the integrons in 1989 they were described as one of major genetic structures which contain one or more gene cassettes located at a specific site $[14,15]$. The basic components [integrase gene (intI), recombination site (attI and attC) and a promoter $(\mathrm{Pc})]$ are found in all types of integrons. Integrons do not have transposition genes therefore they cannot promote their own transfer. But they are transferred with transposons and/or conjugative plasmids. Due to sequences of integrase gene, integrons are classified into five groups [12, 13, 16-18]. Among these classes three of them (class I, class II and class III) are determined to be mostly involved in antibiotic resistance. Class I and class II integrons are defined as the most common and clinically important [13, 17-21].

Integrons are known to be mostly carried in Gram negative bacteria (Acinetobacter, Aeromonas, Enterobacter, Klebsiella, Salmonella, Serratia, E. coli, Vibrio and Campylobacter) which are causative agents of both nosocomial and community acquired infections [22-33]. There are many studies in recent years explained that class I and class II integrons carry many antibiotic resistance genes in Gram positive bacteria such as staphylococci which have become one of the most prevalent resistant pathogens in hospitals during the last decades [7, 9, 34-37]. Both methicillin-resistant S. aureus (MRSA) and coagulase negative staphylococci (MRCNS) which can easily spread by direct or indirect contact among patients and health care personnel are major causes of nosocomial infections all around the world. Quinolone, aminoglycoside and macrolide-lincosamide-streptogramin resistance also widely spreads among staphylococci $[5,6,35,37,38]$.

There is little information about integron bearing staphylococci in all around Europe and Turkey. The aim of this study is to investigate the prevalence of class I and class II integrons in MRSA and MRCNS strains isolated from patients which are the most representative genus of Gram positive bacteria. We also aimed to determine the relation between antibiotic resistance and the presence of integrons. 


\section{MATERIALS AND METHODS}

\subsection{Bacterial strains.}

Sixty four (50.79\%) MRSA and sixty two (49.21\%) MRCNS strains were isolated from various clinical samples (blood, sputum, abscess, swabs, cerebrospinal fluid, etc.) obtained from different patients in İstanbul Yeni Yüzyıl University Medical Faculty, Gaziosmanpaşa Hospital during three years period (2015-18). Repeat isolates were excluded. 110 (87.3\%) out of 126 strains were isolated from hospitalized patients. Identification of strains was performed by automatized systems (VITEK-2). All isolates were tested for susceptibilities to methicillin, gentamycin, ciprofloxacin, erythromycin, vancomycin, teicoplanin, trimethoprim/sulfametoxazole, daptomycin, linezolid, tetracycline, levofloxacin, clindamycin, penicillin by VITEK-2. Strains were kept at $-80^{\circ} \mathrm{C}$ for PCR analysis.

\subsection{DNA extractions.}

Template DNA from staphylococci was prepared from Tryptic soy broth (TSB) cultures grown overnight at $37^{\circ} \mathrm{C}$. An extraction kit (Genemark, Taiwan) was used according to the manufacturer's introductions. To determine the presence of integrons both genomic and plasmid DNAs were investigated.

\subsection{Detection of class $I$ and class II integrons by PCR assays.}

All staphylococci were investigated by multiplex polymerase chain reaction (PCR) for the presence of intI and intII genes [7].

Primers used in this research are shown in Table 1 [7, 39]. Master mix kit (Genemark, Taiwan) was used in PCR. Mixtures ( $25 \mu \mathrm{L}$ last volume) were prepared according to manufacturer's suggestions (Roche, Germany): $5 \mu \mathrm{L}$ master mix, $2 \mu \mathrm{L}$ DNA, $2 \mu \mathrm{L}$ each primer $(1 \mu \mathrm{L}$ for each primer from 10 pmol concentration), and nuclease free water.

Table 1. Primer sequences used in the Polymerase Chain Reaction (PCR) analysis.

\begin{tabular}{|l|l|c|}
\hline Genes & Primer sequences & Amplicon size \\
\hline Class I int F & 5'-CCT CCC GCA CGA TGA TC-3' & \multirow{2}{*}{$280 \mathrm{bp}$} \\
\hline Class I int R & 5'-TCC ACG CAT CGT CAG GC-3' & \\
\hline Class II int F & 5'- TTA TTG CTG GGA TTA GGC-3' & \multirow{2}{*}{233 bp } \\
\cline { 1 - 2 } Class II int R & 5'- ACG GCT ACC CTC TGT TAT C -3' & \\
\hline
\end{tabular}

\subsection{PCR amplification conditions.}

The reaction conditions for PCR amplification were as follows: initial denaturation for 4 min at $94^{\circ} \mathrm{C}$; degradation for $45 \mathrm{sec}$ at $94^{\circ} \mathrm{C}$; annealing for $45 \mathrm{sec}$ at $94^{\circ} \mathrm{C}$; elongation for $55 \mathrm{sec}$ at $72^{\circ} \mathrm{C}$; elongation for $55 \mathrm{sec}$ at $72^{\circ} \mathrm{C}$. These reactions were carried out for 30 cycles (Prima Trio high media thermal cycler, Mumbai, India) [7] and the amplicons were stored at $-20^{\circ} \mathrm{C}$.

\subsection{Electrophoresis.}

The amplified products were separated by gel electrophoresis in a 1.5\% agarose gel stained with ethidium bromide $(0.5 \mu \mathrm{g} / \mathrm{mL})$, visualized under UV light. After that products were electrophoresed for $40 \mathrm{~min}$ under 80 volts with 1 XTBE electrophoretic liquid. The results were visualized and recorded using HiUV MAX transilluminator (HiMedia, India). DNA ladder (Genemark, Taiwan) labeled between 100 1000 bp was used.

All multiplex PCR assays were carried out twice for each strain (plasmid DNA and genomic DNA) to determine if the integrons were associated with chromosome / plasmid or either of them.

\subsection{DNA Sequence Analysis.}

DNA sequences analyses was performed for integron positive DNA samples by using ABI3730 XL Genetic Analyzer device (GATC Biotech AG, Germany).

\subsection{Statistical Analysis.}

Categorical variables were reported as n(\%). Pearson chi-square test and Fisher's exact test were used for comparing categorical variables. SPSS (IBM Corp. Released 2012. IBM SPSS Statistics for Windows, Version 21.0, Armonk, NY: IBM Corp.) was used for statistical analysis and a p value $<0.05$ was considered statistically significant.

$\mathbf{5 0 |}$ | $\mathrm{P}$ a g e 


\section{RESULTS}

3.1. Antimicrobial susceptibilities of MRSA and MRCNS strains

Antimicrobial susceptibilities of 126 methicilin-resistant staphylococci showed that the highest resistance rates were found to penicillin (98.4\%), erythromycin (92.1\%), ciprofloxacin (58\%), tetracycline $(57.2 \%)$, levofloxacin $(54 \%)$ and clindamycin $(50.8 \%)$ which were followed by resistance to gentamycin $(37.3 \%)$, trimethoprim-sulfamethoxazole $(22.22 \%)$ and daptomycin $(1.6 \%)$. None of the tested strains showed resistance to vancomycin, teicoplanin and linezolid.

Comparisons of MRSA and MRCNS strains for antibiotic susceptibility rates were given in Table 2 . The differences between MRSA and MRCNS strains for resistance to gentamicin, ciprofloxacin, trimethoprim-sulfamethoxazole, tetracycline, clindamycin and levofloxacin were found to be statistically significant $(\mathrm{p}<0.05)$ (Table 2).

Table2. Statistically comparison of antibiotic resistance rates in MRSA and MRCNS strains

\begin{tabular}{|c|c|c|c|}
\hline & $\begin{array}{c}\text { MRSA } \\
\mathrm{n}(\%)\end{array}$ & $\begin{array}{c}\text { MRCNS } \\
\text { n }(\%)\end{array}$ & p-value \\
\hline \multicolumn{4}{|l|}{ GN } \\
\hline $\mathrm{S}$ & $47(59.49 \%)$ & $32(40.51 \%)$ & \multirow{2}{*}{$0.011^{\mathrm{a}}$} \\
\hline $\mathrm{R}$ & $17(36.17 \%)$ & $30(63.83 \%)$ & \\
\hline \multicolumn{4}{|c|}{ CIP } \\
\hline $\mathrm{S}$ & $38(71.70 \%)$ & $15(28.30 \%)$ & \multirow[b]{2}{*}{$<0.001^{\mathrm{a}}$} \\
\hline $\mathrm{R}$ & $26(35.62 \%)$ & $47(64.38 \%)$ & \\
\hline \multicolumn{4}{|l|}{$\mathbf{E}$} \\
\hline $\mathrm{S}$ & $4(40 \%)$ & $6(60 \%)$ & \multirow{2}{*}{$0.527^{\mathrm{b}}$} \\
\hline $\mathrm{R}$ & $60(51.72 \%)$ & $56(48.28 \%)$ & \\
\hline \multicolumn{4}{|c|}{ TEC } \\
\hline $\mathrm{S}$ & $64(50.79 \%)$ & $62(49.21 \%)$ & \multirow[b]{2}{*}{-} \\
\hline $\mathrm{R}$ & 0 & 0 & \\
\hline \multicolumn{4}{|c|}{ SXT } \\
\hline $\mathrm{S}$ & $57(58.16 \%)$ & $41(41.84 \%)$ & \multirow{2}{*}{$0.002^{\mathrm{a}}$} \\
\hline $\mathrm{R}$ & $7(25.00 \%)$ & $21(75.00 \%)$ & \\
\hline \multicolumn{4}{|c|}{ VAN } \\
\hline $\mathrm{S}$ & $64(50.79 \%)$ & $62(49.21 \%)$ & \multirow[b]{2}{*}{ - } \\
\hline $\mathrm{R}$ & 0 & 0 & \\
\hline \multicolumn{4}{|c|}{ DAP } \\
\hline $\mathrm{S}$ & $62(50 \%)$ & $62(50 \%)$ & \multirow{2}{*}{$0.496^{\mathrm{b}}$} \\
\hline $\mathrm{R}$ & $2(100 \%)$ & 0 & \\
\hline \multicolumn{4}{|c|}{ LIN } \\
\hline $\mathrm{S}$ & $64(50.79 \%)$ & $62(49.21 \%)$ & \multirow{2}{*}{-} \\
\hline $\mathrm{R}$ & 0 & 0 & \\
\hline \multicolumn{4}{|c|}{ BPEN } \\
\hline $\mathrm{S}$ & $1(50 \%)$ & $1(50 \%)$ & \multirow{2}{*}{$>0.99^{\mathrm{b}}$} \\
\hline $\mathrm{R}$ & $63(50.81 \%)$ & $61(49.19 \%)$ & \\
\hline \multicolumn{4}{|c|}{ TET } \\
\hline $\mathrm{S}$ & $33(61.11 \%)$ & $21(38.89 \%)$ & \multirow{2}{*}{$0.045^{a}$} \\
\hline $\mathrm{R}$ & $31(43.06 \%)$ & $41(56.94 \%)$ & \\
\hline \multicolumn{4}{|c|}{ LEV } \\
\hline $\mathrm{S}$ & $42(72.41 \%)$ & $16(27.59 \%)$ & \multirow{2}{*}{$<0.001^{\mathrm{a}}$} \\
\hline $\mathrm{R}$ & $22(32.35 \%)$ & $46(67.65 \%)$ & \\
\hline \multicolumn{4}{|c|}{ CLIN } \\
\hline $\mathrm{S}$ & $39(62.90 \%)$ & $23(37.10 \%)$ & \multirow{2}{*}{$0.007^{a}$} \\
\hline $\mathrm{R}$ & $25(39.06 \%)$ & $39(60.94 \%)$ & \\
\hline
\end{tabular}

$\overline{\text { Data are expressed as } n(\%) .{ }^{a} \text { : Chi-Square Test, }{ }^{b} \text { : Fisher's Exact Test }}$

$\mathrm{S}$ : susceptible; R: resistant; $\mathrm{p}$ value $<0.05$ is accepted as significant difference.

GN: gentamycin; CIP: ciprofloxacin; E: erythromycin; SXT: trimethoprim/sulfametoxazole; DAP: daptomycin; TET: tetracycline; LEV: levofloxacin; CLIN: clindamycin; P: penicillin 
Two of 126 strains were found to be resistant to only penicillin. Among 124 multiple resistant staphylococci, there are 27 resistance patterns (Table 3). The most prevalent resistance pattern was ciprofloxacin - erythromycin - penicillin - tetracycline resistance (CEPT) (38 strains). It was followed by erythromycin - penicillin resistance (EP) (19 strains) (Table 3).

Table 3. Antibiotic resistance patterns of staphylococci and their relation to the presence of integrons.

\begin{tabular}{|c|c|c|}
\hline Antibiotic resistance pattern & $\begin{array}{l}\text { number of } \\
\text { strains }\end{array}$ & $\begin{array}{l}\text { number of classI and/or classII } \\
\text { integron bearing strains }\end{array}$ \\
\hline CIP, E, P, TET (CEPT) & 38 & 6 \\
\hline $\mathrm{E}, \mathrm{P}(\mathrm{EP})$ & 19 & 3 \\
\hline E, P, TET, CLIN & $\mathbf{1 0}$ & 1 \\
\hline GN, CIP, E, SXT, P, TET, LEV, CLIN & 9 & 0 \\
\hline E, P, TET & 8 & 2 \\
\hline CIP, E, P, LEV, CLIN & 6 & 3 \\
\hline CIP, E, P, LEV & 4 & 0 \\
\hline GN, CIP, E, P, LEV, CLIN & 4 & 1 \\
\hline E, P, CLIN & 3 & 1 \\
\hline $\mathrm{SXT}, \mathrm{P}$ & 3 & 1 \\
\hline GN, CIP, E, SXT, P, LEV & 3 & 0 \\
\hline CIP, E, P, LEV & 2 & 0 \\
\hline $\mathrm{E}, \mathrm{SXT}, \mathrm{P}$ & 2 & 0 \\
\hline GN, E, P,TET & 1 & 0 \\
\hline CIP, SXT, P, TET, LEV & 1 & 0 \\
\hline GN, CIP, E, P, LEV & 1 & 0 \\
\hline E, TET & 1 & 0 \\
\hline CIP, E, SXT, P, LEV, CLIN & 1 & 1 \\
\hline GN, E, P, TET & 1 & 0 \\
\hline E, SXT, P, CLIN & 1 & 0 \\
\hline GN, CIP, E, P, LEV, CLIN & 1 & 0 \\
\hline CIP, E, SXT, P, LEV & 1 & 0 \\
\hline P, TET, CLIN & 1 & 0 \\
\hline GN, P, CLIN & 1 & 0 \\
\hline CIP, P, TET, LEV & 1 & 0 \\
\hline GN, CIP, P, TET, LEV, CLIN & 1 & 0 \\
\hline GN, E, P,TET & 1 & 0 \\
\hline
\end{tabular}

GN: gentamycin; CIP: ciprofloxacin; E: erythromycin; SXT: trimethoprim/sulfametoxazole; DAP: daptomycin; TET: tetracycline; LEV: levofloxacin; CLIN: clindamycin; P: penicillin

\subsection{Detection of class 1 and class 2 Integrons}

Among 126 staphylococci 11 (8.7\% - 4 MRSA and 7 MRCNS) were shown to carry class I integron; whereas 7 MRCNS (5.5\%) were class II positive. Both of class I and class II integrons were detected to possess in four MRSA (3.2\%) (Figure 1, Figure 2). None of the MRCNS isolates have both of class I and class II integrons.

Class I integron in three intI positive MRSA were found to be encoded on both plasmid and chromosome; another four MRSA harboured class I integron only on chromosome. However all intII gene was encoded only on plasmids.

In a total of $22(17.5 \%)$ classI and/or classII integron bearing strains only $20(90.9 \%)$ were found to be isolated from hospitalized patients. On the other hand it was shown that, 16 (72.7\%) out of 22 integron bearing strains were isolated from blood cultures. 


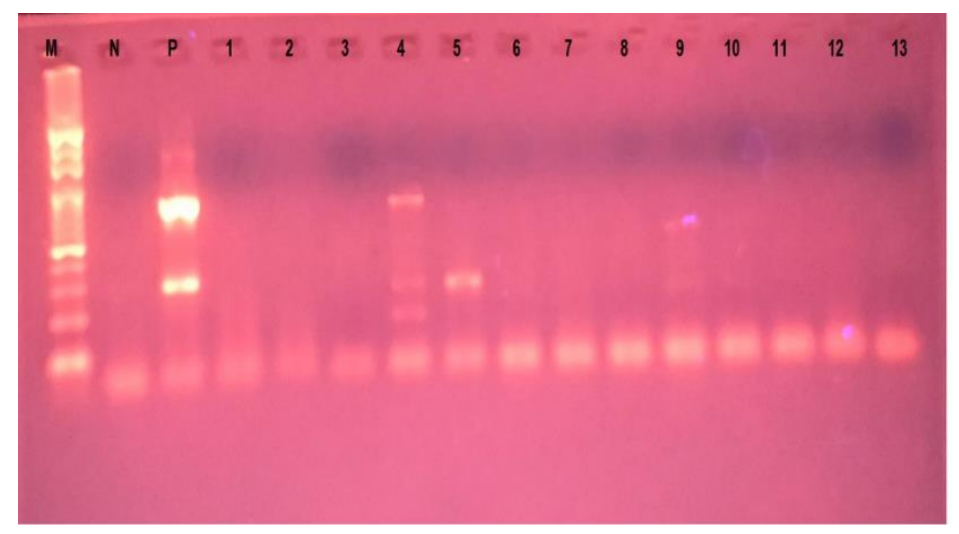

Figure 1. Agarose gel image of the of intI and intII genes amplified by the multiplex PCR. (M: Marker; N: Negative Control, P: Positive Control (intI--280 bp and intII-788 bp), 1-3: negative samples, 4: intI and intII positive sample, 5: intI positive sample, 6-13: negative samples

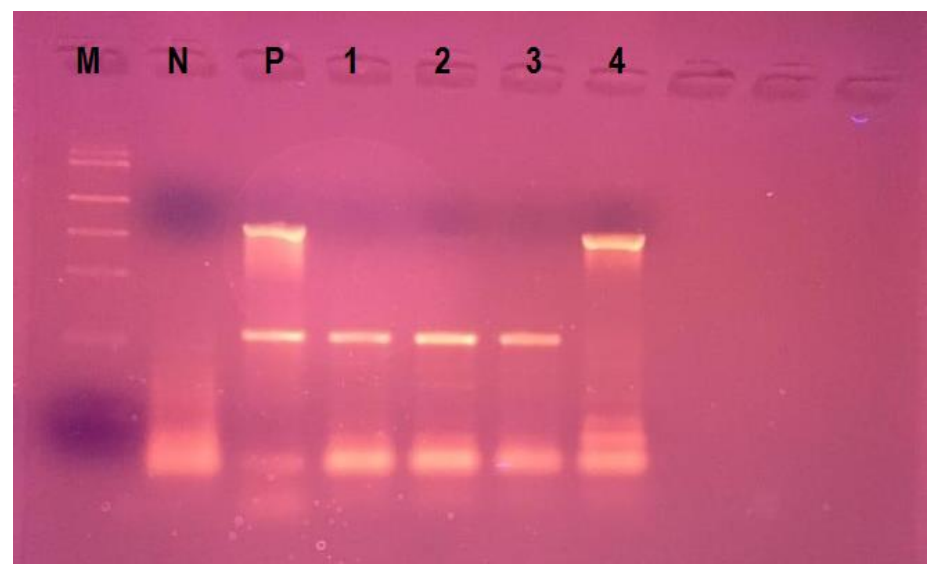

Figure 2. Agarose gel image of the of intI and intII genes amplified by the multiplex PCR. (M: Marker; N: Negative Control, P: Positive Control (intI--280 bp and intII-788 bp), 1-3: intI positive samples, 4 : intII positive sample

\subsection{Antibiotic susceptibilities of integron bearing strains.}

The highest resistance rates in integron bearing 22 strains were detected to penicillin $(100 \%)$, erythromycin $(86.4 \%)$, clindamycin $(50 \%)$ and ciprofloxacin $(50 \%)$. The percentage of resistance to levofloxacin $(45.5 \%)$, tetracycline $(45.5 \%)$, trimethoprim/sulfametoxazole $(13.7 \%)$, gentamicin $(9.1 \%)$ and daptomycin (0) ranged from 0 to $45.5 \%$.

There was no statistically significant relation between presence of integrons and resistance to each of antibiotics $(\mathrm{p}>0.05)$.

In two most prevalent multiple resistance pattern groups (CEPT and EP) six strains of the group CEPT and three strains of the group EP were found to carry classI and/or classII integron. Other integron harboring strains were included in various resistance pattern groups. On the other hand, two penicillin resistant strains were also found to carry class I integron.

\section{DISCUSSION}

Multiple resistant - epidemic MRSA and MRCNS strains are the most common pathogens particularly in nosocomial infections [5, 6, 34, 35, 37, 38]. Emergent multiple antibiotic resistance is the major problem causing treatment failure $[1,2,6,38]$. It is well known that, integrons play a major role in rapid evolution and dissemination of antibiotic resistance due to their ability of capture different gene cassettes encoding resistance to various antibiotics [9, 17]. In the present study, we aimed to investigate the presence of class I and II integrons in MRSA and MRCNS strains; and the relationship between integrons 
and antibiotic resistance.

Investigations focusing on presence of integrons in staphylococci and other Gram positive bacteria are very few and they were mostly originated from specific geographic regions such as China and Middle East. Class I integrons were first described in Corynebacterium glutamicum in 1998; and since then integron related antibiotic resistance genes were shown to be present in different Gram positive bacteria [40, 41]. In 1999, class I integron was detected in an Enterococcus faecalis [33] and the first definition of class I integron in Staphylococcus isolated from poultry litter was reported in 2004 [42]. Moreover in 2010 four Streptococcus strains defined to carry class I integron by $\mathrm{Xu} \mathrm{Z}$ et al.; and same authors also reported the first definition of class II integron in Enterococcus faecium in China [43].

In 2007, 16 (53\%) of 30 clinical and environmental isolates of $S$. aureus were found to be positive for class I integron [9]. In 2008, same authors reported the presence of class I integron in 30 clinical multiple resistant MRCNS strains. The relation between high resistance rates and integron bearing was not emphasized [8]. They also announced the first isolation of class I integron in clinical nosocomial MRSA strains 44]. In 2011, Xu Z et al. have examined 471 methicillin resistant staphylococci for presence of class I-III integrons and they found that 122 strains beared class I integron [45].

Ren C et al. (2013) first described a multiplex PCR method capable of detecting class I-III concurrently in clinical $S$. aureus strains; and with their new method they informed that, none of the $S$. aureus strains were shown to carry class III integron and class I and class II integrons were mostly encoded on plasmid DNA (class I integron 51.1\%, class II integron 12.78\%) rather than genomic DNA (class I integron $39.4 \%$, class II integron 5.8\%). The authors also examined the relations between antibiotic resistance rates and presence of integrons. They also reported that multiple antibiotic resistance rates associated with the presence of classI and classII integrons [7].

Yahaghi E. et al. (2014) reported that, $2(1 \%)$ out of 200 clinical $S$. aureus strains were found to carry class I integron [46]. In another study clinically isolated $S$. lugdunensis strains were found to harbor class I $(25 \%)$ and class II integrons (7\%); whereas class III integron was not detected in any of them. No significant associations were found between presence of integrons (class I and class II) and resistance to each antibiotic; but multiple antibiotic resistances has related to the presence of integrons [38].

Mostafa M et al. (2015) have shown that, 101 (73\%) of 139 clinical $S$. aureus strains were found to carry class I integron whereas class II integron positivity was lower (35\%). They found that there are significant relations between resistance to gentamycin, spectinomycin, azithromycine, cefrtriaxone and the presence of integrons [47].

In a study conducted by Hosseini et al. has identified class I (39.6\%) and class II (3.7\%) integrons' presence in clinical $S$. aureus strains. Similar to previous studies the authors emphasized that class III integron was not found; and there were no statistically significant relation between integron presence and antibiotic resistance [48].

In Turkey, investigations focusing on presence of integrons in Gram positive bacteria are very few. Only reported study in literature to our knowledge is conducted by Güney AK et al. They reported that no class I integron was detected in any of MRCNS or MRSA strains examined [6].

In general consistent with previous studies antibiotic resistance rates were high to tetracycline, penicillin, gentamycin, clindamycin, ciprofloxacin, trimethoprim/sulfamethoxasole and streptomycin [6, 7, 47]. In the present study, we found that resistance rates to gentamicin, ciprofloxacin, trimethoprimsulfamethoxazole, tetracycline, clindamycin and levofloxacin were significantly higher in MRCNS rather than MRSA. Besides, both MRSA and MRCNS strains were shown to have two classes of integrons (8 MRSA and 14 MRCNS strains). To our knowledge this is the first study showing the presence of integrons and their relations to antibiotic resistance examined in MRSA and MRCNS strains in Turkey. In this study, the prevalence of integron bearing staphylococci is consistent with previous studies. Although there was no statistically significant relation between resistance rates and presence of integrons; our results showed an important data about the distribution of integrons among staphylococci.

\section{Acknowledgments}

The authors would like to thank undergraduate students Esra Eroğlu, Firdevs Deniz Camadan and Merve Oral for their technical support. This research was supported by TUBITAK (The Scientific and Technological Research Council of Turkey) (Project No: 2209-a / 2018).

Ethical approval has been provided from Istanbul Yeni Yüzyıl University Medical Faculty Research Ethics Committee. Meeting Date: 28.02.2017 No: 013. 


\section{References}

1. Giedraitiené, A. et al. (2011). Antibiotic resistance mechanisms of clinically important bacteria. Medicina. 47, 137-146.

2. Džidić, S. et al. (2008). Antibiotic resistance mechanisms in bacteria: biochemical and genetic aspects. Food Technol Biotechnol. 46, 11-21.

3. Du, X. et al. (2005). Characterization of class 1 integrons-mediated antibiotic resistance among calf pathogenic Escherichia coli. FEMS microbiology let. 245, 295-298.

4. Wright, GD. et al. (2005). Bacterial resistance to antibiotics: enzymatic degradation and modification. Adv Drug Deliv Rev. 57, 1451-70.

5. Procop, GW. et al. (2017). Koneman's Color Atlas and Textbook of Diagnostic 7th ed. Philadelphia: Wolters Kluwer.

6. Guney, AK. et al. (2014). A study on class I integrons and antimicrobial resistance among clinical staphylococci isolates from a Turkish hospital. Clinical Microbiology: Open Access. 3, 1-4.

7. Ren, C. et al. (2013). Analysis of the effect of integrons on drug-resistant Staphylococcus aureus by multiplex PCR detection. Mol Med Rep. 7, 719-724.

8. Xu, Z. et al. . (2008) Integron-bearing methicillin-resistant coagulase-negative staphylococci in South China, 2001-2004. FEMS Microbial. Lett. 278, 223-230.

9. Xu, Z. et al. (2007). Nosocomial infection caused by class 1 integron-carrying Staphylococcus aureus in a hospital in South China. Clin. Microbial. Infec. 13, 980-984.

10. Naghoni, A. (2010). High prevalence of integron-mediated resistance in clinical isolates of Salmonella enterica. Jpn. J. Infect. Dis. 63, 417-421.

11. Levesque, C. et al. (1995). PCR mapping of integrons reveals several novel combinations of resistance genes. Antimicrob Agents Chemother. 39, 185-191.

12. Collis, CM. et al. (2002). Characterization of the class 3 integron and site-specific recombination system it determines. J Bacteriol. 184, 3017-26.

13. Heir, E. (2004). Prevalence and characterization of integrons in blood culture Enterobacteriaceae and gastrointestinal Escherichia coli in Norway and reporting of a novel class 1 integron-located lincosamide resistance gene. Ann Clin Microbiol Antimicrob. 3, 12.

14. Stokes HW and Hall RM (1989). A novel family of potentially mobile DNA elements encoding a site-specific gene-integration function: integron. Mol Microbiol. 3, 1669-83.

15. Nield, BS et al. (2001). Recovery of new integron classes from environmental DNA. FEMS Microbiol Lett. 195, 59-65.

16. Hansson, K. et al. (2002). IntI2 integron integrase in Tn7. J Bacteriol. 184, 1712-21.

17. Ploy, MC. et al. (2000). Integrons: an antibiotic resistance gene capture and expression system. Clin Chem Lab Med. 38, 483-487

18. Li, Y. et al. (2017). Microbial pathogenicity and virulence mediated by integrons on Gram-positive microorganisms. Microb Patho. 111, 481-486.

19. Fonseca, EL. et al. (2005). Class 1 integrons in Pseudomonas aeruginosa isolates from clinical settings in amozon region, Brazil. FEMS Immunol Med Microbiol. 44, 303-309. 
20. Maguire, AJ. et al. (2001). Rapid screening technique for class 1 integrons in Enterobacteriaceae and nonfermenting Gram-negative bacteria and its use in molecular epidemiology. Antimicrob Agents Chemother. 45, 1022-29.

21. Weldhagen, GF. (2004). Integrons and $\beta$-lactamases- a novel perspective on resistance. Int J Antimicrob Agents. 23, 556-562.

22. Koeleman, JGM. et al. (2001). Identification of epidemic strains of Acinetobacter baumannii by integrase gene PCR. J Clin Microbiol. 39, 8-13.

23. L'Abee-Lund, TM. and Sorum, H. (2001), Class 1 integrons mediate antibiotic resistance in the fish pathogen Aeromonas salmonicida worldwide. Microb Drug Resist. 7, 263-272.

24. Crowley, D et al. (2002). Molecular epidemiology of cystic fibrosis-linked Burkholderia cepacia complex isolates from three national referral centres in Ireland. J Appl Microbiol. 92, 992-1004.

25. Gibreel, A. and Skold, O. (2000). An integron cassette carrying dfr1 with 90-bp repeat sequences located on the chromosome of trimethoprim-resistant isolates of Campylobacter jejuni. Microb Drug Resist. 6, 91-98.

26. Norskov-Lauritsen, N. et al. (2001), Clonal origin of aminoglycoside-resistant Citrobacter freundii isolates in a Danish county. J Med Microbiol, 50, 636-641.

27. Clark, NC. et al. (1999). Detection of a streptomycin/ spectinomycin adenylyltransferase gene $(\operatorname{aadA})$ in Enterococcus faecalis. Antimicrob Agents Chemother. 43, 157-160.

28. Centron, D. and Roy, PH. (2002). Presence of a group II intron in a multiresistant Serratia marcescens strain that harbors three integrons and a novel gene fusion. Antimicrob Agents Chemother. 46, 1402-1409.

29. Falbo, V. et al. (1999). Antibiotic resistance conferred by a conjugative plasmid and a class I integron in Vibrio cholerae O1 El or strains isolated in Albania and Italy. Antimicrob Agents Chemother. 4, 693-696.

30. Arakawa, Y. et al.(1995) A novel integron-like element carrying the metallo-b-lactamase gene blaIMP. Antimicrob Agents Chemother. 39, 1612-15.

31. Correia, M. et al.(2003). Molecular characterization of a new class 3 integron in Klebsiella pneumoniae. Antimicrob Agents Chemother. 47, 2838-43.

32. Nemergut, DR. et al.(2008). Insights and inferences about integron evolution from genomic data. BMC Genomics. 9, 1-12

33. Clark, CA. et al. (2000). The Vibrio cholerae O1 chromosomal integron. Microbiol. 146, 26052612 .

34. Shi, L. (2006). Unnoticed spread of class 1 integrons in gram-positive clinical strains isolated in Guangzhou, China. Microbiol Immunol. 50 463-467.

35. Hiramatsu, K. et al. (2014). Multi-drug-resistant Staphylococcus aureus and future chemotherapy. J Infect Chemother. 20, 593-601.

36. Kaneko, J. and Kamio, Y. (2004). Bacterial two-component and heteroheptameric pore-forming cytolytic toxins: structures, pore-forming mechanism, and organization of the genes. Biosci Biotechnol Biochem. 68, 981-1003.

37. Lowy, FD. (2013). Methicillin-resistant Staphylococcus aureus: where is it coming from and where is it going? JAMA Intern Med. 173(21), 1978-79. 
38. Pirbonyeh, N. (2019). Integron related resistance in new emerged Staphylococcus lugdunensis infection in burn patients. J Burn Care Res. 1-17.

39. Goldstein, C. et al. (2001). Incidence of class 1 and 2 integrases in clinical and commensal bacteria from livestock, companion animals, and exotics. Antimicrob Agents Chemother. 45(3), 723-726

40. Deng, Y, et al. (2015). Antimicrobial resistance investigation on Staphylococcus Strains in a local Hospital in Southern China, 2001-2010. Microb Drug Resist. 21, 102-104.

41. Nesvera, J. et al. (1998). An integron of class 1 is present on the plasmid pCG4 from Grampositive bacterium Corynebacterium glutamicum. FEMS Microbiol Lett. 169, 391-395.

42. Nandi, S. (2004). Gram-positive bacteria are a major reservoir of Class 1 antibiotic resistance integrons in poultry litter. Proceed. National Aca Scien. 101(18), 7118-22.

43. Xu, Z. et al. (2010). First report of class 2 integron in clinical Enterococcus faecalis and class 1 integron in Enterococcus faecium in South China. Diag Microbial Infec Dis. 68, 315-317.

44. Xu, Z. et al. (2008). First confirmation of integron-bearing methicillin-resistant Staphylococcus aureus. Curr Microbiol. 57. 264-268.

45. Xu, Z. et al. (2011). Class 1 integron in staphylococci. Mol Biol Rep. 38,5261-79.

46. Yahaghi, E. et al. (2014). Detection of class I integrons in Staphyloacoccus aureus isolated from clinical samples. Iran Red Cres Med Jour, 16, 11.

47. Mostafa, M. et al. (2015). Variability in gene cassette patterns of class 1 and 2 integrons associated with multi drug resistance patterns in Staphylococcus aureus clinical isolates in Tehran-Iran. BMC Microbiol. 15, 152.

48. Hosseini SM, et al. (2019). The First Report of Prevalence of Class 1-3 Integrons in Clinical Isolates of Staphylococcus aureus in Southwestern Iran: A Multicenter Study. Jundishapur J Microbiol. 12:11. 